\title{
Isolation and epithelial co-culture of mouse renal peritubular endothelial cells
}

\author{
Ye Zhao ${ }^{1}$, Hong Zhao ${ }^{1,2}$, Yun Zhang ${ }^{1,3}$, Tania Tsatralis ${ }^{1}$, Qi Cao ${ }^{1}$, Ya Wang ${ }^{1}$, Yiping Wang ${ }^{1}$, Yuan Min Wang ${ }^{4}$, \\ Steve I Alexander ${ }^{4}$, David C Harris $^{1}$ and Guoping Zheng ${ }^{1 *}$
}

\begin{abstract}
Background: Endothelial-mesenchymal transition (EndoMT) has been shown to be a major source of myofibroblasts, contributing to kidney fibrosis. However, in vitro study of endothelial cells often relies on culture of isolated primary endothelial cells due to the unavailability of endothelial cell lines. Our recent study suggested that peritubular endothelial cells could contribute to kidney fibrosis through EndoMT. Therefore, successful isolation and culture of mouse peritubular endothelial cells could provide a new platform for studying kidney fibrosis. This study describes an immunomagnetic separation method for the isolation of mouse renal peritubular endothelial cells using anti-CD146 MicroBeads, followed by co-culture with mouse renal proximal tubular epithelial cells to maintain endothelial phenotype.

Results: Flow cytometry showed that after isolation and two days of culture, about $95 \%$ of cells were positive for endothelial-specific marker CD146. The percentage of other cells, including dendritic cells (CD11c) and macrophages (F4/80), was less than 1\%. Maintenance of endothelial cell phenotype required vascular endothelial growth factor (VEGF) and co-culture with mouse proximal tubular epithelial cells.

Conclusion: In this study, we established a method for the isolation of mouse renal peritubular endothelial cells by using immunomagnetic separation with anti-CD146 MicroBeads, followed by co-culture with mouse renal proximal tubular epithelial cells to maintain phenotype.
\end{abstract}

Keywords: Peritubular endothelial cells, Tubular epithelial cells, CD146, Co-culture, Vascular endothelial growth factor

\section{Background}

Endothelial cells have been found to be a major source of myofibroblasts via endothelial mesenchymal transition (EndoMT), causing fibrosis in kidney and other organs [1-4]. In one key study, Zeisberg and colleagues [4] showed in three mouse models including unilateral ureteral obstructive nephropathy (UUO), Alport disease and streptozotocin-induced diabetic nephropathy, that around $30 \%$ to $50 \%$ of fibroblasts formed in the kidneys coexpressed the endothelial marker CD31 and the fibroblast/ myofibroblast markers fibroblast specific protein-1 (FSP-1) and/or $\alpha$-smooth muscle actin ( $\alpha$-SMA). Endothelial lineage tracing using Tie2-Cre;R26R-stop-EYFP transgenic mice in the UUO model [4] and in streptozotocin-induced diabetic nephropathy [5] further confirmed the presence

\footnotetext{
* Correspondence: guoping.zheng@sydney.edu.au

${ }^{1}$ Centre for Transplant and Renal Research, Westmead Millennium Institute,

The University of Sydney, Sydney, NSW, Australia

Full list of author information is available at the end of the article
}

of EndoMT-derived fibroblasts. However, elucidation of molecular mechanisms of EndoMT involving glomerular or peritubular endothelial cells relies largely on in vitro studies using primary isolated human or mouse endothelial cells. Such studies are limited by the loss of phenotype that occurs in those primary endothelial cells in culture after a limited number of passages.

Renal endothelial cells include glomerular endothelial cells, peritubular endothelial cells and vascular endothelial cells. Although it is generally accepted that endothelial cells contribute to fibroblast formation in kidney, the contribution of different renal endothelial cells has not been defined. Previous studies examining EndoMT in renal fibrosis were mostly focused on glomerular endothelial cells, not surprisingly using the well-established method for isolation of glomerular endothelial cells [6-9]. By immunofluorescence staining of kidney sections of mice with UUO, co-localization of the mesenchymal marker $\alpha$-SMA and endothelial marker CD31 or VE- 
cadherin was observed predominantly outside glomeruli, suggesting that the interstitial peritubular rather than glomerular endothelial cells play the major role, at least in the UUO model. To date, however, a method for isolation of peritubular endothelial cells of high purity has not been described [10]. For example, the method described by Mcginn et al. [8] may isolate lymphatic and vascular endothelial cells.

Primary endothelial cells are susceptible to phenotypic change in culture; a co-culture system was, therefore, developed to mimic the in vivo micro-environment in the kidney with its key interactions between renal tubular epithelial cells and adjacent endothelial cells. Tasnim et al. [10] described interactions by which human renal glomerular endothelial cells improved the stability of the human renal tubular cell phenotype while glomerular endothelial cell phenotype was also well-maintained by tubular epithelial cells. However, such a system may not be applicable to the interaction between peritubular endothelial cells and tubular epithelial cells in vivo.

Here we describe a method for isolation of primary mouse renal peritubular endothelial cells (MRPEC) and co-culture with mouse renal proximal tubular epithelial cells. Our results show that separation with anti-CD146 MicroBeads resulted in peritubular endothelial cells of high purity. Further, co-culture with mouse renal proximal tubular epithelial cells maintained the phenotype of isolated peritubular endothelial cells, thus providing a stable in vitro model for investigating the role of peritubular endothelial cells in kidney diseases.

\section{Methods}

Animals

Male BALB/c mice (6 week old) were purchased from Australian Research Council and experiments were performed in accordance with protocols approved by Animal Ethics Committee of Western Sydney Local Health District.

\section{Separation of tubular fraction from kidney cortex}

Mouse kidney tubular fractions were obtained from the kidney cortex of BALB/c mice using established methods adapted from Doctor et al. [11]. Kidneys were perfused in situ via the aorta with $20 \mathrm{ml}$ phosphate buffered saline (PBS; Lonza; Walkersville, MD, USA) containing 80U/ml heparin to remove blood from anesthetized mice. Kidney capsule was removed by peeling with forceps. Freshly isolated kidneys were placed in ice-cold Dulbecco's Modified Eagle's Medium mixed with Ham's F12 (DMEM/F12; 1:1 ratio; Gibco Life Technologies; Grand Island, NY, USA) on a petri dish. The kidney was sliced coronally and homogenized by mincing into $1 \mathrm{~mm}^{3}$ to $2 \mathrm{~mm}^{3}$ pieces. The homogenized kidney cortex tissue pieces were resuspended and mixed in $7.5 \mathrm{ml}$ of collagenase type IV solution (Table 1) and incubated at $37^{\circ} \mathrm{C}$ in a gentle shaking water bath for $15 \mathrm{~min}$. The suspension was homogenized by pipetting 5 to 10 times through a sterile transfer pipette followed by addition of $1 \mathrm{ml}$ of fresh collagenase type IV solution. This process was repeated 2-4 times. About $40 \mathrm{ml}$ fresh ice-cold DMEM/F12 was then added into the collagenase digestion solution and the suspension was centrifuged at $200 \times \mathrm{g}$ for $2 \mathrm{~min}$. The pellet was resuspended and washed in $10 \mathrm{ml}$ of fresh ice-cold DMEM/F12 and centrifuged at $150 \times \mathrm{g}$ for $2 \mathrm{~min}$ at $4^{\circ} \mathrm{C}$. Densitygradient centrifugation of the pellet was then performed by resuspension in $25 \mathrm{ml}$ of $45 \%$ ( $\mathrm{vol} / \mathrm{vol}$ ) sterile Percoll solution (Table 2) in $50 \mathrm{ml}$ centrifugation tubes and centrifugation at $5525 \times \mathrm{g}$ for $30 \mathrm{~min}$ at $4^{\circ} \mathrm{C}$ (without braking). After centrifugation, the tubule fractions were collected from the top layer of the Percoll solution $(5 \mathrm{ml}$ of the top layer). The tubule fraction was washed once in $20 \mathrm{ml}$ ice-cold DMEM/F12 medium at $300 \times \mathrm{g}$ for $5 \mathrm{~min}$ at $4^{\circ} \mathrm{C}$ and resuspended for further experiments.

\section{Isolation of proximal tubular epithelial cells from tubule fraction}

The pellet was resuspended in $\mathrm{K} 1$ medium [DMEM: HAM's F12; 1:1 vol/vol; Gibco Life Technologies) supplemented with $25 \mu \mathrm{g} / \mathrm{ml}$ of epithelial growth factor (EGF; Sigma Chemical Co.; St. Louis, MO, USA), $25 \mu \mathrm{M}$ HEPES (Invitrogen; Carlsbad, CA, USA), hormone mixture (Table 3) and 5\% fetal calf serum (FCS; Invitrogen)]. Proximal tubular epithelial cells were obtained by direct culture after removing peritubular endothelial cells.

\section{Isolation of peritubular endothelial cells from tubule fraction}

The tubule fractions were further digested with $0.025 \%$ trypsin or collagenase type IV solution for 10 to $15 \mathrm{~min}$ at $37^{\circ} \mathrm{C}$ to obtain single cell suspensions. After digestion, ice-cold PBS was added to the cell suspension followed by filtering sequentially through a $70-\mu \mathrm{m}$ and a $40-\mu \mathrm{m}$ cell strainer. The filtered cell suspensions were then centrifuged at $300 \times \mathrm{g}$ for $2 \mathrm{~min}$ at $4^{\circ} \mathrm{C}$. The pellet was resuspended in MicroBeads resuspension buffer [prepared in PBS, containing $0.5 \%$ BSA (Sigma Chemical Co.) and $2 \mathrm{mM}$ EDTA (Life Technologies)] and centrifuged again at $300 \times \mathrm{g}$ for $3 \mathrm{~min}$ at $4^{\circ} \mathrm{C}$. The cell pellets obtained

\begin{tabular}{lll} 
Table $\mathbf{1}$ Components of collagenase type IV solution \\
\hline Collagenase D solution & $\begin{array}{l}\text { Volume or } \\
\text { concentration }\end{array}$ & Supplier \\
\hline DMEM/F12 medium & $7.5 \mathrm{ml}$ & $\begin{array}{l}\text { Gibco Life Technologies; } \\
\text { Grand Island, NY, USA }\end{array}$ \\
Collagenase type IV & $1 \mathrm{mg} / \mathrm{ml}$ & $\begin{array}{l}\text { Sigma Chemical Co.; } \\
\text { St Louis, MO, USA }\end{array}$ \\
Deoxyribonuclease & $0.1 \mathrm{mg} / \mathrm{ml}$ & $\begin{array}{l}\text { Sigma Chemical Co. } \\
\text { Bovine serum albumin (BSA) }\end{array}$ \\
\hline
\end{tabular}


Table 2 Percoll solution $(25 \mathrm{ml})$

\begin{tabular}{|c|c|c|}
\hline Percoll solution & Volume & Supplier \\
\hline DMEM/F12 medium & $12.5 \mathrm{ml}$ & $\begin{array}{l}\text { Gibco Life Technologies; } \\
\text { Grand Island, NY, USA }\end{array}$ \\
\hline Percoll (45\%, vol./vol.) & $11.25 \mathrm{ml}$ & Gibco Life Technologies \\
\hline $20 \mathrm{X}$ concentrated PBS & $0.5625 \mathrm{ml}$ & AMRESCO; Solon, OH, USA \\
\hline MilliQ water & $0.5625 \mathrm{ml}$ & $\begin{array}{l}\text { Roche Applied Science; } \\
\text { Penzberg, Upper Bavaria, Germany }\end{array}$ \\
\hline $1 \mathrm{M}$ HEPES & $80.5 \mu \mathrm{l}$ & Gibco Life Technologies \\
\hline
\end{tabular}

from 2 kidneys were resuspended in $1 \mathrm{ml}$ MicroBeads resuspension buffer and incubated with anti-mouse CD16/32 antibody (Fc-blocking) for $15 \mathrm{~min}$ to block non-specific binding. The cell pellets were then magnetically labeled with CD146 (LSEC) MicroBeads (MACS; Miltenyi Biotechnology; Bergisch Gladbach, Germany) and separated according to the manufacturer's instructions. Specifically, cells were incubated with CD146 MicroBeads and then passed through a magnetic field. Peritubular cells were collected and cultured in the prewarmed endothelial cell medium (ScienCell; Carlsbad, CA, USA) in fibronectin (Sigma Aldrich) pre-coated Transwell plates. Purity of the isolated peritubular endothelial cells was determined by immunofluorescent staining and flow cytometric analysis. Before FACS staining, the beads were detached from the cells using the MultiSort Release Reagent (MACS), which enzymatically removes the MicroBeads [Additional file 1].

\section{Flow cytometry analysis (FACS)}

Adherent endothelial cells were detached from culture flasks with trypsin/EDTA (Life Technologies), washed and resuspended in PBS (Gibco Life Technologies) containing $1 \%$ FCS. Cells $\left(10^{6}\right.$ cells $\left./ \mathrm{ml}\right)$ were then incubated with respective FITC-, PE- or APC-conjugated monoclonal antibodies for $20 \mathrm{~min}$ at $4^{\circ} \mathrm{C}$. Fluorescence antibody-labeled cells were then washed twice in cold PBS with 1\% FCS and analyzed using a flow cytometer (BD Bioscience; San Jose, CA, USA).

\section{Antibodies}

Antibodies for flow cytometry were as follows: anti-mouse F4/80 antigen Alexa Fluor ${ }^{\circ}$ 488, anti-mouse Lyve-1 Alexa Fluor $^{\odot}$ 488, anti-mouse CD31 (PECAM-1) APC, antimouse CD45 PE, anti-mouse CD11c Alexa Fluor 488 (eBioscience; San Diego, CA, USA), PE-anti-mouse CD146, Alexa Fluor ${ }^{\circ} 488$ anti-mouse panendothelial cell antigen (Biolegend; San Diego, CA, USA), FITC-anti-mouse FSP-1/ S100A4 (LifeSpan BioSciences; Seattle, WA, USA) and anti-mouse PDGFR- $\beta$ Alexa Fluor 488 (BD Bioscience). For isotype antibody controls, rat IgG2a $\kappa$ Alexa Fluor ${ }^{\circ}$ 488 (eBioscience) was used for F4/80, rat IgG1 к Alexa Fluor $^{\oplus} 488$ (eBioscience) was used for Lyve-1, FSP-1 and PDGFR- $\beta$, rat IgG2a $\kappa$ APC (eBioscience) was used for CD31, rat IgG2a $\kappa$ PE (eBioscience) was used for CD45, Armenian hamster IgG Alexa Fluor ${ }^{\oplus} 488$ (eBioscience) was used for CD11c, and PE rat IgG2a, $\mathrm{k}$ (Biolegend) was used for CD146, and Alexa Fluor 488 rat IgG2a, $\mathrm{K}$ (Biolegend) was used for PV-1.

Primary antibodies for immunofluorescence were as follows: rabbit polyclonal anti-VE-cadherin (1:200; Alexis Biochemicals; Farmingdale, NY, USA), rabbit polyclonal anti-FSP-1/S100A4 (1:400; Merck Millipore; Billerica, MA, USA), rat monoclonal anti-CD11c (1:200; eBioscience), rat monoclonal anti-F4/80 (1:200; eBioscience), purified rat anti-CD73 (1:50; BD Bioscience), rabbit monoclonal antiPDGFR- $\beta$ (Abcam; Cambridge, UK), and mouse monoclonal anti-actin, $\alpha$-smooth muscle (Sigma Chemical Co.). For isotype antibody controls, rat IgG2a $\mathrm{k}$ Purified (eBioscience) was used for VE-cadherin and F4/80, rabbit IgG (Invitrogen) was used for FSP-1 and PDGFR- $\beta$, Armenian hamster IgG purified (eBioscience) was used for CD11c, Purified Mouse IgG1, к (BD Bioscience) was used for CD73, and mouse IgG2a, $\kappa$ (Biolegend) was used for $\alpha-S M A$.

Table 3 Components of hormone mixture in $\mathrm{K} 1$ medium

\begin{tabular}{|c|c|c|c|}
\hline Ingredient & Supplier & Stock solution & Vol./wt. of addition \\
\hline Insulin & Sigma Chemical Co., St. Louis, MO, USA & Powder & $\begin{array}{l}50 \mathrm{mg} \text { dissolved in } 10 \mathrm{ml} \text { of HBSS/HEPES* } \\
\text { and small amount of } \mathrm{NaOH}\end{array}$ \\
\hline Prostaglandin $\mathrm{E}_{1}$ & Cayman Chemical, Ann Arbor, MI, USA & 0.5 mg/ml (in EtOH) & $25 \mu l$ \\
\hline 3,3,5-triiodothyro-nine & Sigma Chemical Co. & 16.9 mg/ml (0.026 M) (in EtOH) & $\begin{array}{l}2 \mu \mathrm{l} \text { of stock added to } 10 \mathrm{ml} \mathrm{HBSS} / \mathrm{HEPES}^{*} \\
\text { then used in } 100 \mu \mathrm{l} \text { aliquots }\end{array}$ \\
\hline Transferrin & Sigma Chemical Co. & Powder & $50 \mathrm{mg}$ \\
\hline Sodium selenite & Sigma Chemical Co. & $0.173 \mathrm{mg} / \mathrm{ml}\left(10^{-6} \mathrm{M}\right)$ in HBSS/HEPES & $100 \mu \mathrm{l}$ \\
\hline Hydrocortisone & Sigma & 0.18 mg/ml (in EtOH) & $1 \mathrm{ml}$ \\
\hline
\end{tabular}

The hormone mixture was brought to a final volume of $100 \mathrm{ml}$ with $\mathrm{HBSS} / \mathrm{HEPES}$, and aliquoted into $5 \mathrm{ml}$ or $10 \mathrm{ml}$ portions and stored at $-80^{\circ} \mathrm{C}$.

*HBSS/HEPES = Hanks Balanced Salt Solution without calcium and without magnesium (Gibco, Life Technologies; Grand Island, NY, USA)/1\% HEPES. 
Secondary antibodies for immunofluorescence were as follows: fluorescent-conjugated secondary anti-rabbit Alexa Fluor 488 (1:600; Invitrogen), anti-mouse Alexa Fluor 546 (1:600; Invitrogen) and anti-rat Alexa Fluor 488 (1:600; Invitrogen).

\section{Cell culture}

The primary mouse peritubular endothelial cells isolated using MicroBeads were cultured in endothelial cell medium containing VEGF (Sigma-Aldrich; St. Louis, MO, USA) $(2.5 \mu \mathrm{g} / \mathrm{ml}$ to $5 \mu \mathrm{g} / \mathrm{ml})$ according to a previously described method [6]. The endothelial cell medium contained $93 \%$ of basal medium (ScienCell), 5\% fetal bovine serum (FBS; ScienCell), 1\% endothelial cell growth supplement (ECGS; ScienCell) and 1\% penicillin/ streptomycin (P/S; ScienCell). Cells were maintained at $37^{\circ} \mathrm{C}$ in $5 \% \mathrm{CO}_{2}$ and incubated overnight in plates precoated with fibronectin in endothelial cell medium, nonadherent cells were removed and medium was changed. Then medium was changed every 2-3 days. Experiments with mono-cultures and co-cultures were performed in fibronectin precoated 6-well Transwell plates (BD Bioscience) with polyester inserts (pore size $0.4 \mu \mathrm{m}$ ) with endothelial cell medium. The proximal tubular epithelial cells from tubule fractions were incubated overnight in plates in $\mathrm{K} 1$ medium at $37^{\circ} \mathrm{C}$ with $5 \% \mathrm{CO}_{2}$, non-adherent cells were removed and medium was changed. Then medium was changed every 2-3 days. In the co-culture system, peritubular endothelial cells were placed in the bottom chamber of Transwell plates while proximal tubular epithelial cells were placed in polyester inserts and cultured in endothelial cell medium.

\section{Immunofluorescence}

For immunofluorescent staining, cells were seeded on glass coverslips in 6-well culture plates and cultured until they reached $50 \%$ to $60 \%$ confluence. Cells were then washed with PBS, fixed with absolute methanol for $10 \mathrm{~min}$ at $-20^{\circ} \mathrm{C}$, and blocked with $2 \%$ BSA (Sigma Chemical Co.) for $1 \mathrm{~h}$ at room temperature. The coverslips were incubated for $1 \mathrm{~h}$ at room temperature with primary antibodies against endothelial cell marker VE-cadherin, fibroblast cell marker FSP-1, dendritic cell marker CD11c, macrophage cell marker F4/80, perivascular fibroblast marker CD73, or pericyte specific marker PDGFR- $\beta$. After washing in PBS and distilled water, cells were incubated with fluorescentconjugated secondary antibody for $40 \mathrm{~min}$ at room temperature in the dark. The coverslips were then washed in PBS and distilled water and counterstained with 4',6-diamidino-2-phenylindole (DAPI) (Invitrogen) for $5 \mathrm{~min}$. After washing in PBS and distilled water, the coverslips were mounted with fluorescence mounting medium (Dako; Glostrup, Denmark) and subjected to fluorescence microscopy. Isotype controls of corresponding secondary antibodies were used as negative controls.

\section{Semiquantitative assessment of immunofluorescent staining}

A minimum of 10 consecutive fields of immunofluorescent images were taken from each slide of stained MRPECs with total cell count of at least 2000 cells. Results were obtained from duplicate slides of a minimum three independent experiments. The number of positive staining cells was counted according to the number of nuclei counterstained by DAPI (blue).

\section{Statistical analysis}

Results from at least three independent experiments were expressed as mean \pm SEM. Statistical significance was evaluated using a two-tail t-test for comparison between two groups. A value of $P<0.05$ was considered statistically significant.

\section{Results}

Anti-CD146 MicroBeads isolation of peritubular endothelial cells from the tubule fraction of kidney cortex Following isolation using anti-CD146 MicroBeads, the peritubular endothelial cells were assessed by FACS analysis. The FACS results show that before magnetic separation, the percentage of CD146-positive cells was around $8 \%$ (Figure 1A, left panel). The percentage of CD146-positive cells was around 38\% after magnetic separation (Figure 1A, middle panel). To improve purity, we used Fc-blocking and typsinisation. Fc-blocking was used in our isolation of peritubular endothelial cells with anti-CD146 (rat anti-mouse) MicroBeads as described [12] to avoid non-specific binding. To obtain single-cell suspensions from MicroBeads isolation, the tubule fraction underwent digestion with optimized concentrations of trypsin to avoid co-sorting of non-endothelial cells by sorting of endothelial-non-endothelial cell aggregates. The FACS results showed that Fc-blocking and trypsin digestion increased the percentage of CD146 positive endothelial cells from 39\% (Figure 1A, middle panel) to $45 \%$ (Figure 1A, right panel).

\section{MicroBeads concentration optimization and kidney perfusion to increase purity of isolated peritubular endothelial cells}

After MicroBeads (1:20) isolation, 15\% of cells were CD45+ (Figure 1B, middle panel), suggesting contamination with blood cells. Therefore, we performed kidney perfusion before digestion to remove blood cell. The peritubular endothelial cell purity thus was increased to $70 \%$ and CD45 positive cells reduced to around $1 \%$ (Figure 1B, right panel). To further improve the purity, the MicroBeads to cell suspension ratio was optimized. 


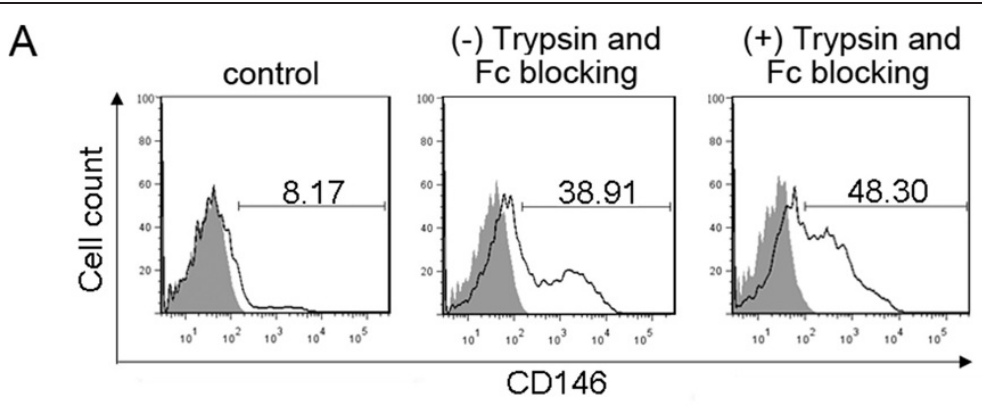

B

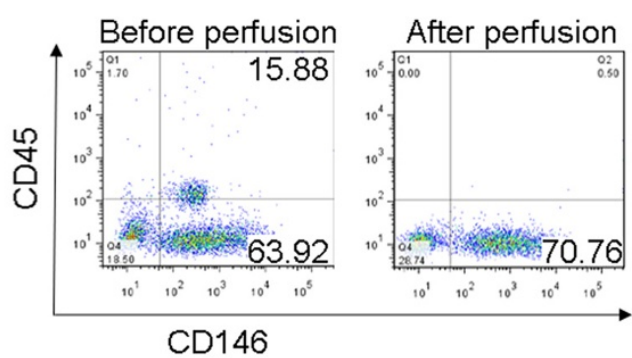

C
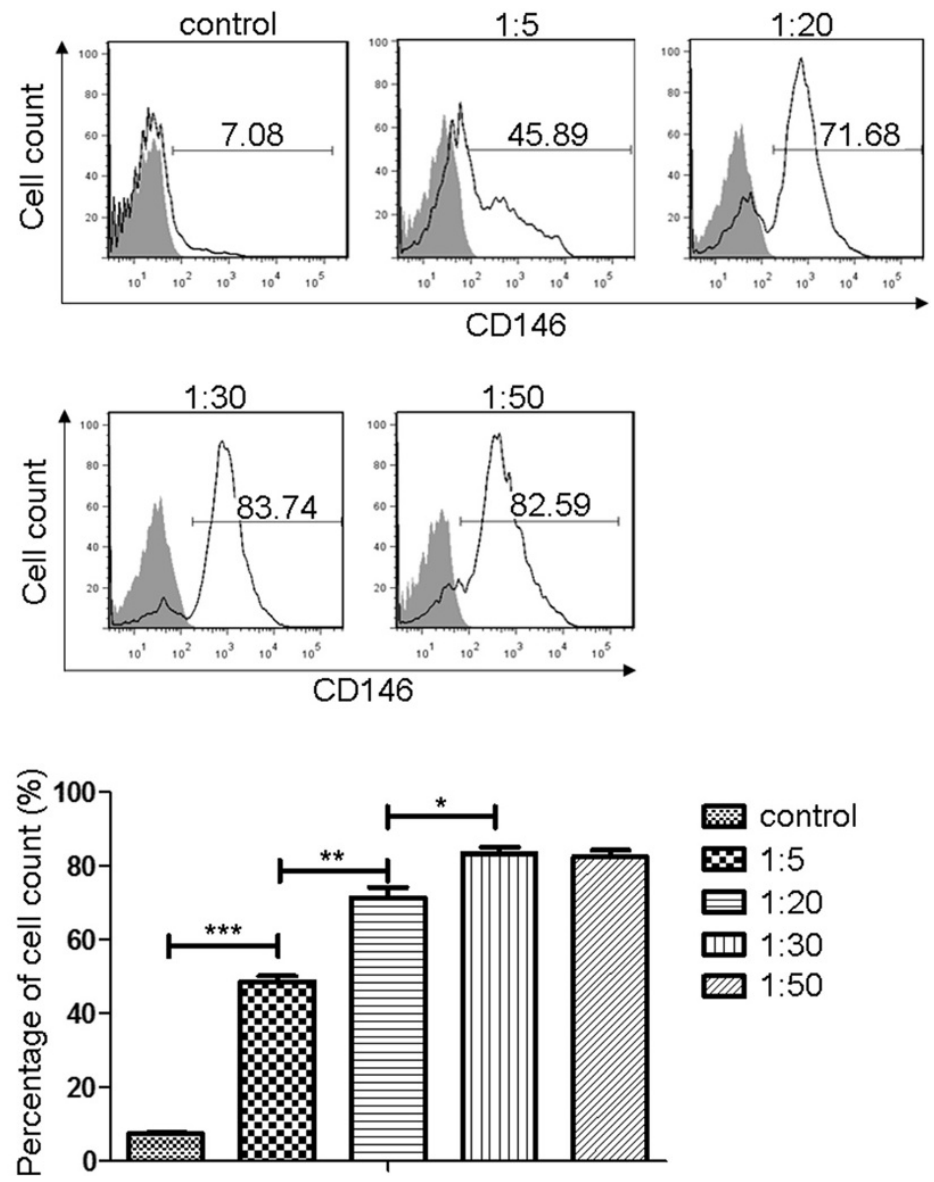

control

죠 1:5

回:20

메 1:30

m $1: 50$

Figure 1 (See legend on next page.) 
(See figure on previous page.)

Figure 1 Flow cytometric analysis of peritubular endothelial cells isolated by MicroBeads. (A) Flow cytometry analysis of CD146-PE positive endothelial cells before (left panel), after MicroBeads isolation (middle panel), and after MicroBeads isolation with Fc-blocking and trypsin digestion (right panel). Shaded areas are respective isotype controls. (B) Flow cytometry analysis of blood cell (CD45) contamination when CD146-PE positive endothelial cells were isolated with MicroBeads (1:20) with (right panel) or without kidney perfusion (left panel). Shaded areas are respective isotype controls. (C) After perfusion, CD146-PE positive cells before (top left panel) and after MicroBeads isolation at different MicroBeads concentration: 1:5 (top middle panel), 1:20 (top right panel), 1:30 (bottom left panel) and 1:50 (bottom right panel). Shaded areas are respective isotype controls. (G) Statistical analysis of each individual experiment $(\mathrm{N}=3)$. After modification of kidney perfusion and optimization of MicroBeads concentration, the purity of CD146 positive cells increased significantly from $7.5 \pm 0.3$ (before separation) to as high as $83.3 \pm 1.7$. Data are expressed as mean \pm SEM with $\mathrm{N}=3$ for each experimental group. ${ }^{*} P<0.05$, ${ }^{*} P<0.01,{ }^{* *} P<0.001$.

After kidney perfusion, when MicroBeads to cell suspension ratio was changed from 1:5 to $1: 30$ (Figure $1 C$, top panels), the purity of CD146 positive endothelial cells was increased to $>80 \%$ (Figure $1 \mathrm{C}$, bottom left panel) while the purity by further dilution at a ratio of 1:50 remained at the same level (Figure $1 \mathrm{C}$, bottom right panel). So the final ratio of MicroBeads to cell suspension used was 1:30.

\section{2 days of culture after MicroBeads isolation to increase purity of peritubular endothelial cell}

After incubation with the optimal concentration of MicroBeads (1:30) and following immunomagnetic separation, the percentage of CD146-positive cells remained stable at about $80 \%$. Magnetically isolated peritubular endothelial cells were cultured for two days and then analyzed with antibodies for different cell surface markers to determine the purity of endothelial cells and possible contamination of other cells by FACS. The peritubular endothelial cells were incubated with antibodies for lymphatic vessel endothelial cell marker Lyve-1, dendritic cell marker CD11c, hematopoietic cell marker CD45, macrophage cell marker F4/80, fibroblast cell marker FSP-1, pericyte cell marker PDFGR- $\beta$, and endothelial cell markers CD146 and CD31. To distinguish from glomerular endothelial cells, PV-1 was used as peritubular endothelial marker [13]. The FACS results showed that the respective percentage for other cell type was less than $1.83 \%$ (Figure 2A). The percentage of CD31 positive cells was 78\% and that of CD146 positive cells was $92 \%$ (Figure $2 \mathrm{~B}$ ). These results could be due to CD31 being trypsin sensitive [12] while CD146 is trypsin resistant. However, the percentage of contaminating cells could potentially be higher if their surface markers were trypsin sensitive. The results after 2 days culture suggested that culturing of isolated peritubular endothelial cells further improved their purity, possibly through separation of adherent endothelial cells from other non-adherent cells. Over 2 million cells were obtained from each mouse.

\section{Immunofluorescence staining to show high purity of isolated mouse renal peritubular endothelial cells (MRPECs)}

The high purity of isolated MRPECs shown by FACS analysis was further confirmed with immunofluorescent staining. After two days of culture, immunofluorescent staining of isolated MRPECs showed that all cells were VE-cadherin positive and few cells were FSP-1 positive (Figure 3A, B). Immunofluorescent staining for CD11c and F4/80 also suggested that no dendritic or macrophage cells were present (Figure $3 \mathrm{C}, \mathrm{E}$ ). To confirm the absence of pericyte contamination, cells were stained for CD73 and PDGFR- $\beta$. Results showed that almost none

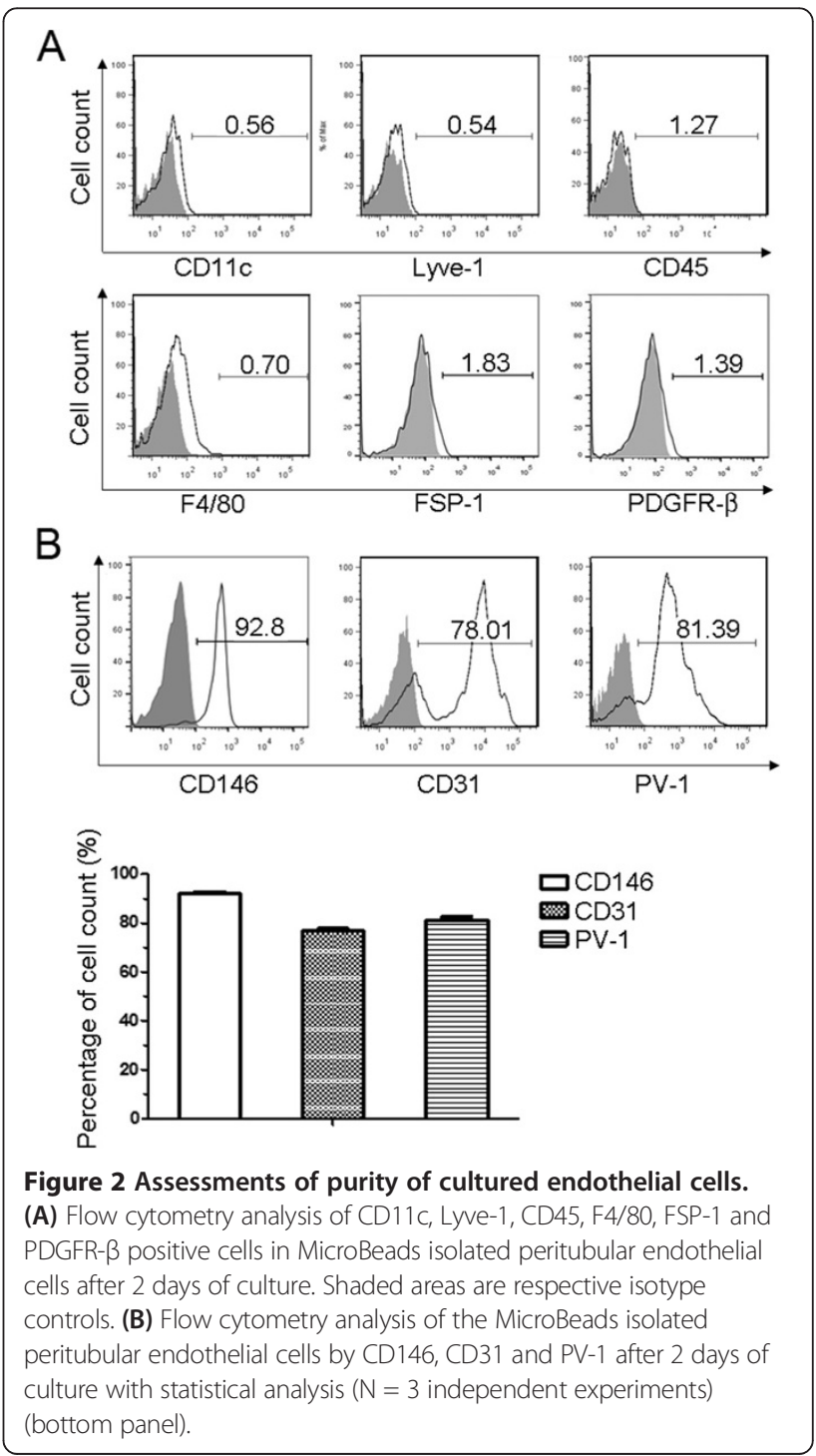




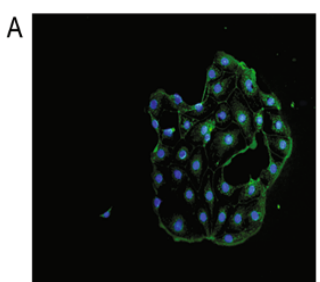

VE-cadherin

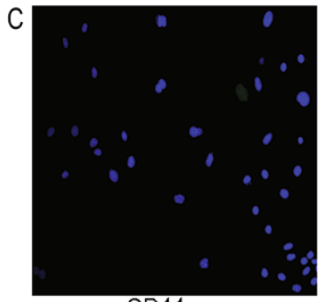

CD11c

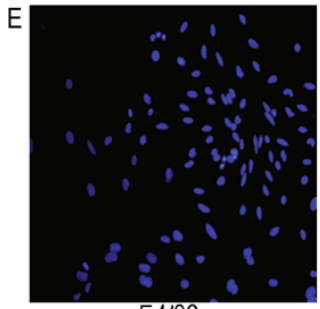

$\mathrm{F} 4 / 80$

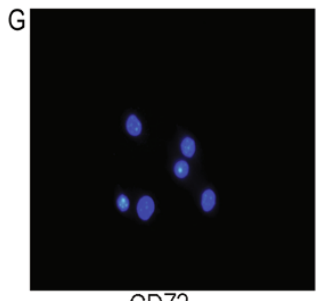

$\mathrm{CD} 73$

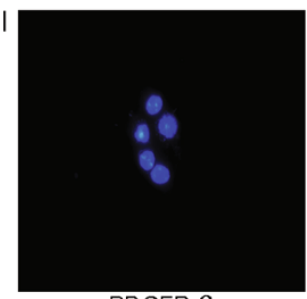

PDGFR- $\beta$

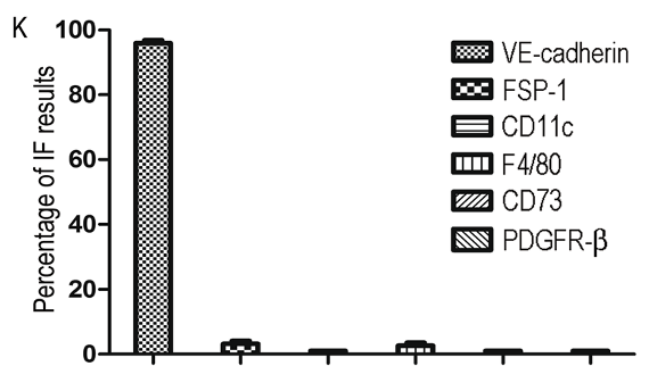

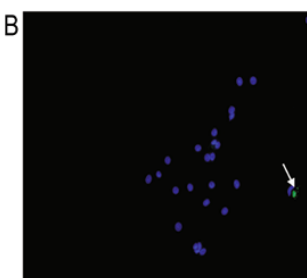

FSP-1
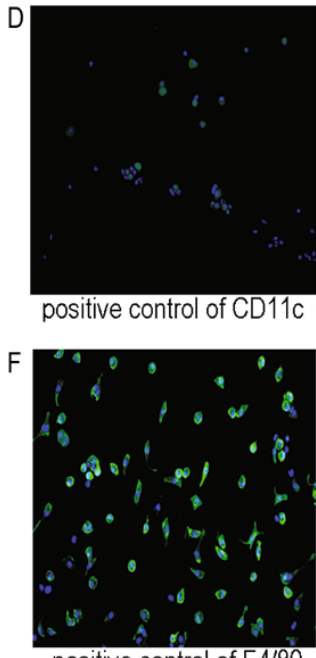

positive control of F $4 / 80$
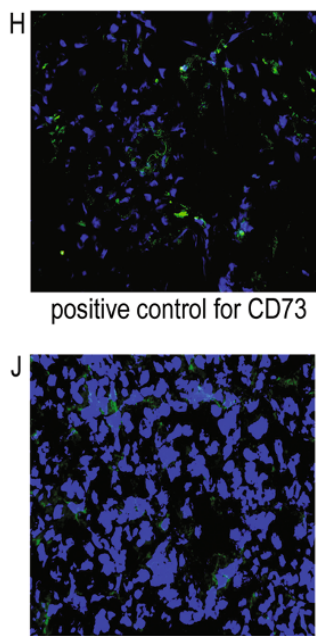

positive control for PDGFR- $\beta$ positive control for CD73

of the cells expressed CD73 or PDGFR- $\beta$ (Figure 3G, I). Therefore, taken together the FACS analysis and immunofluorescent results showed that MRPECs of high purity were successfully isolated from mouse renal tissue.

\section{Co-culture with proximal tubular epithelial cells and VEGF} to prevent phenotypic change of isolated peritubular endothelial cells

Immunofluorescence staining of MRPECs cultured for 2, 4 and 6 days showed VE-cadherin positive staining. However, a large number of cells were also $\alpha$-SMA positive after 2 days of culture, suggesting that the cells were undergoing phenotypic change (Figure 4A). To maintain MRPEC endothelial phenotype, vascular endothelial growth factor (VEGF) was added to the culture medium and MRPECs were co-cultured with mouse proximal tubular epithelial cells (MPTECs) which were obtained via direct culture of tubule fractions (Figure 4B). FSP-1 and $\alpha$-SMA were used as markers for fibroblasts [4]. While FSP-1 (Figure 4C, top panels) staining was low in all groups, $\alpha$-SMA staining co-localized with VE-cadherin (Figure 4C, bottom panels) significantly diminished in culture of peritubular endothelial cells after treatment with VEGF and especially when combined in co-culture with proximal tubular epithelial cells (Figure 4C, D). Statistical analysis showed the effect of co-culture of MRPECs with MPTECs was minimal as compared to mono-culture of MRPECs without any treatment (Figure 4D). However, $\alpha$-SMA expression decreased significantly when the cells were treated with VEGF (from $35.0 \pm 3.6$ to $14.3 \pm 3.0$, $P<0.05)$. When the cells were co-cultured with MPTECs and treated with VEGF, the number of $\alpha$-SMA positive cells was the lowest among all groups (from $35.0 \pm 3.6$ to $5.3 \pm 1.5, P<0.001$ ) (Figure 4D). This result demonstrated that co-culture with MPTECs and VEGF treatment is an effective method for maintaining isolated MRPECs. 

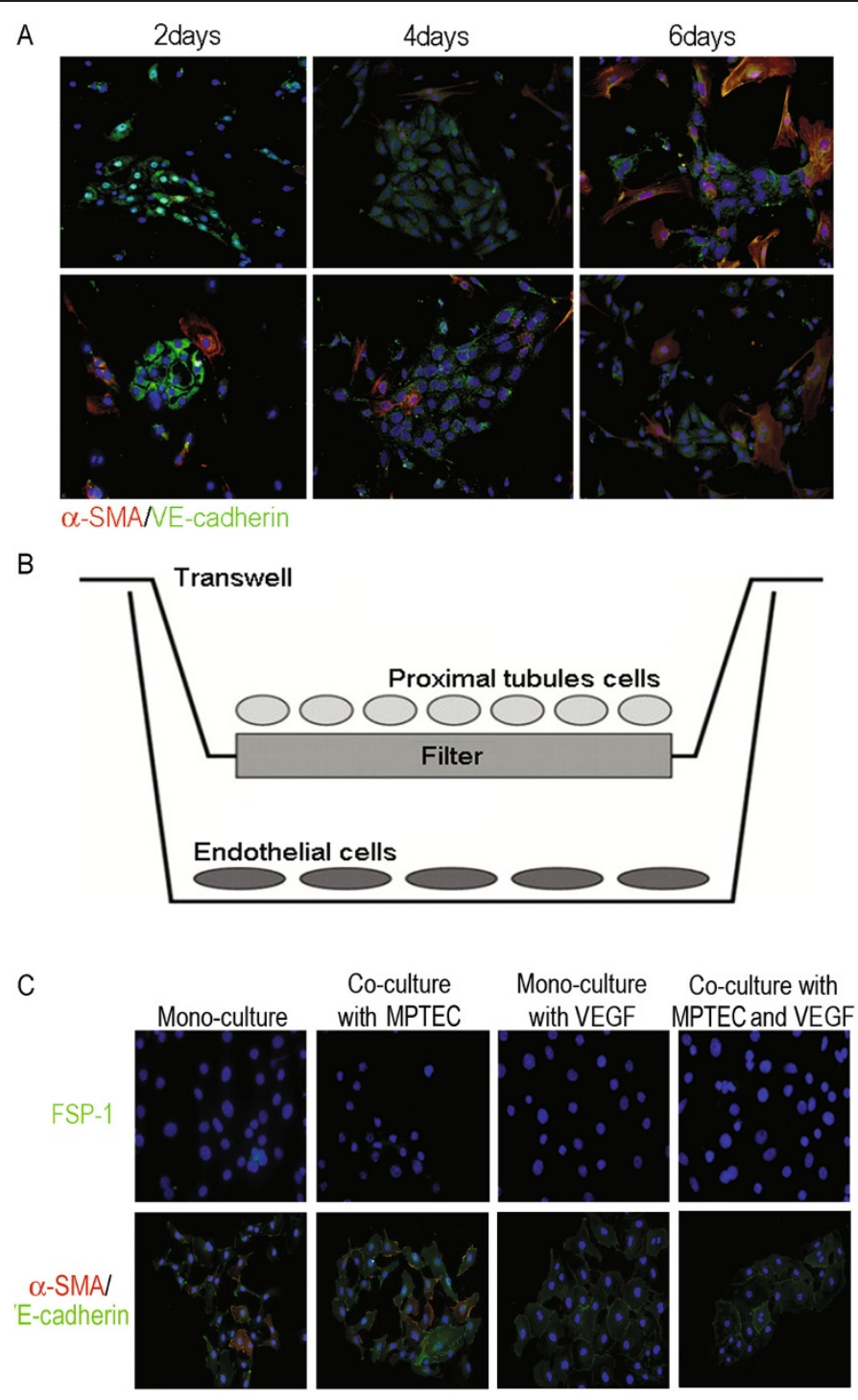

D

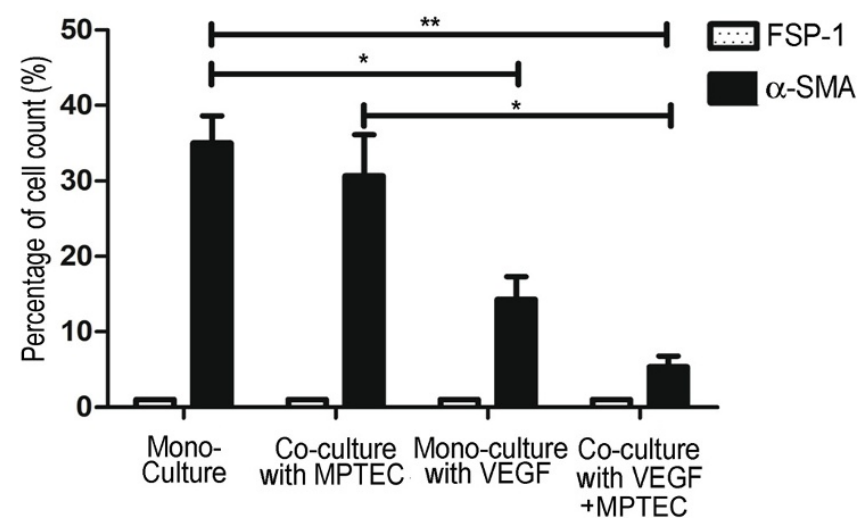

Figure 4 (See legend on next page.) 
(See figure on previous page.)

Figure 4 Primary mouse renal peritubular endothelial cell performance in mono- and co-culture with mouse proximal tubular epithelial cell over 6 days. (A) Co-localization of VE-cadherin (green) and a-SMA (red) positive cells cultured in MPRECs mono-cultures without VEGF. (B) Peritubular endothelial cell and proximal tubular epithelial cell co-culture. In the co-culture system, isolated peritubular endothelial cells were placed in the bottom chamber of fibronectin pre-coated Transwell plates and proximal tubular epithelial cells were seeded onto the polyester inserts (pore size $0.4 \mu \mathrm{m}$ ). Endothelial cell medium was used for the co-culture. (C) After 6 days, primary mouse renal peritubular endothelial cell performance in mono- and co-culture in the presence or absence of VEGF was assessed by FSP-1 (green, top panels) staining and co-localization of a-SMA (red) and VE-cadherin (green, bottom panels). Orange represents a-SMA and VE-cadherin double positive areas. (D) Statistical analysis of primary mouse renal peritubular endothelial cell performance after cells were cultured for 6 days. FSP-1 (green) and a-SMA (red) expression in MRPECs was quantified and expressed as a percentage of positive stained cells against total cells. Images presented are representative of at least 5 independent replicate experiments. Data are expressed as mean \pm SEM with $N=5$ for each group. ${ }^{*} P<0.05$, ${ }^{* *} P<0.01$ vs. respective control. Original magnification was $x 200$. Cells in this figure were counterstained with DAPI to visualize nuclei (blue).

\section{Discussion}

Anti-CD146-conjugated MicroBeads have been used for isolation of endothelial cells from liver [14]. Anti-CD31 was previously used in isolation of endothelial cells. However, pericytes are also positive for CD31. In addition, endothelial surface expression of CD31 could be decreased by trypsin digestion during isolation as demonstrated in our current study. Therefore, we used for the first time anti-CD146 MicroBeads to isolate peritubular endothelial cells. Our results showed that the percentage of CD146-positive cells was as high as 92\% while that of CD31-positive cells was up to $80 \%$. To separate peritubular endothelial cells from pericytes, which may also be positive for CD146, we isolated peritubular cells using an established method for high-purity tubules isolation [15] to avoid pericyte contamination. Isolated peritubular endothelial cells stained negatively for the pericyte markers CD73 and PDGFR- $\beta$ [16], while they were positive for VE-cadherin.

To improve the purity of cells isolated from tubular fractions with trypsin-EDTA digestion, treatment of the suspensions with Fc-blocking reagents (mouse Ig) is needed to avoid contamination with the other types of cells that express Fc receptor. Marelli-Berg et al. [12] showed that treatment with Fc-blocking reagents is essential for high specificity and efficiency during positive selection, particularly when using antibodies derived from a closely related species (e.g. rat anti-mouse).

Enzymatic digestion, particularly with trypsin-EDTA, has been reported by Marelli-Berg and colleagues to be capable of lowering surface level of CD31 [12]. They showed that much higher levels of CD31 could be detected on endothelial cells as compared to those endothelial cells after treatment with trypsin-EDTA solution to detach them from the culture flasks. This may account for the difference between CD31 and CD146 staining (80\% vs. 92\%) for isolated peritubular endothelial cells. However, trypsin treatment is necessary for preparation of single cell suspensions. In addition, we optimized the trypsin concentration to minimize the influence on other cell surface markers.
Renal peritubular endothelial cells are, however, susceptible to undergoing phenotypic change in culture. Manual removal of phenotype-changed cells [8] is technically difficult to optimize. A co-culture system was, therefore, developed to mimic the in vivo microenvironment of the kidney in which the interactions between renal tubular epithelial cells and adjacent endothelial cells are vital for survival of peritubular endothelial cells [10]. VEGF was used previously to aid maintenance of endothelial cell phenotype [6]. Our results proved that VEGF can help MRPECs maintain their phenotype, possibly because VEGF has profound effects on endothelial cells and stimulates their survival and proliferation, as well as vasculogenesis and angiogenesis $[17,18]$. Furthermore, co-culture with proximal tubular epithelial cells increased the stability of the endothelial phenotype beyond that achieved by incubation with VEGF alone. It is possible that renal epithelial cells and endothelial cells formed a micro-environment that positively affects both cell types and promotes survival and proliferation.

The potentially critical interactions between epithelial and endothelial cells and soluble factors in the microenvironment have been described in a study by Tasnim and Zink [10]. They showed that co-culture with proximal tubular epithelial cells stimulated glomerular endothelial cells to express increased amounts of hepatocyte growth factor (HGF) and VEGF. In addition, endothelial cells also secreted increased amounts of TGF- $\beta 1$ and its antagonist $\alpha 2$-macroglobulin (A2M) in the presence of proximal tubular epithelial cells. A2M balanced the effects of TGF- $\beta 1$ and the long-term maintenance of renal epithelia was improved in the presence of HGF and VEGF $[19,20]$. Here we described a co-culture of tubular epithelial cells with peritubular endothelial cells. Although the mechanism remains to be explored, peritubular rather than glomerular endothelial cells are the endothelial cells that interact with tubular epithelial cells in vivo in kidney. Thus, the co-culture system better reflects the physiological environment of peritubular endothelial cells. 


\section{Conclusions}

We have successfully isolated peritubular endothelial cells from mouse renal tissue with high purity and maintained their phenotype in a co-culture system. The methods of peritubular endothelial cell isolation and co-culture established in this study should provide an in vitro model for investigation the role of peritubular endothelial cells in kidney fibrosis and all other tubulointerstitial kidney diseases.

\section{Additional file}

Additional file 1: Procedures for isolation of MRPECs.

\section{Abbreviations}

A2M: a2-macroglobulin; CKD: Chronic kidney disease; DAPI: 4',6-diamidino-2phenylindole; ECGS: Endothelial cell growth supplement; EndoMT: Endothelial mesenchymal transition; HGF: Hepatocyte growth factor; FBS: Fetal bovine serum; FCS: Fetal calf serum; FSP-1: Fibroblast specific protein-1; HGF: Hepatocyte growth factor; MRPEC: Mouse renal peritubular endothelial cell; MPTEC: Mouse proximal tubular epithelial cell; PBS: Phosphate buffered saline; P/S: Penicillin/streptomycin; $a-S M A$ : $a$-smooth muscle actin; UUO: Unilateral ureteral obstruction; VE-cadherin: Vascular endothelial cadherin; VEGF: Vascular endothelial growth factor.

\section{Competing interests}

The authors declare that they have no competing interests.

\section{Authors' contributions}

$Y Z$ performed all experiments and drafted the manuscript. $H Z, Y Z$ and $\Pi T$ participated in the animal work. QC, YW, YMW, and YW participated in cell culture and flow cytometry. SIA, DCH and GZ conceived of the study, and participated in its design and coordination and helped to draft the manuscript. All authors read and approved the final manuscript.

\section{Acknowledgments}

We thank the Flow Cytometry Facility of Westmead Millennium Institute and Westmead Animal House. This work was supported by the National Health and Medical Research Council (NHMRC) project Grant 632688, Australia.

\section{Author details}

${ }^{1}$ Centre for Transplant and Renal Research, Westmead Millennium Institute, The University of Sydney, Sydney, NSW, Australia. ${ }^{2}$ Department of Biochemistry and Molecular Biology, Shanxi Medical University, Taiyuan, PR China. ${ }^{3}$ Experimental Centre of Science and Research, The First Clinical Hospital of Shanxi Medical University, Taiyuan, PR China. ${ }^{4}$ Centre for Kidney Research, Children's Hospital at Westmead, Sydney, NSW, Australia.

Received: 4 March 2014 Accepted: 16 October 2014

Published online: 30 November 2014

\section{References}

1. Hashimoto N, Phan SH, Imaizumi K, Matsuo M, Nakashima H, Kawabe T, Shimokata K, Hasegawa Y: Endothelial-mesenchymal transition in bleomycin-induced pulmonary fibrosis. Am J Respir Cell Mol Biol 2010, 43(2):161-172

2. Kizu A, Medici D, Kalluri R: Endothelial-mesenchymal transition as a novel mechanism for generating myofibroblasts during diabetic nephropathy. Am J Pathol 2009, 175(4):1371-1373.

3. Li J, Bertram JF: Review: endothelial-myofibroblast transition, a new player in diabetic renal fibrosis. Nephrology (Carlton) 2010, 15(5):507-512.

4. Zeisberg EM, Potenta SE, Sugimoto $H$, Zeisberg M, Kalluri R: Fibroblasts in kidney fibrosis emerge via endothelial-to-mesenchymal transition. J Am Soc Nephrol 2008, 19(12):2282-2287.

5. Li J, Qu X, Bertram JF: Endothelial-myofibroblast transition contributes to the early development of diabetic renal interstitial fibrosis in streptozotocininduced diabetic mice. Am J Pathol 2009, 175(4):1380-1388.
6. Akis N, Madaio MP: Isolation, culture, and characterization of endothelial cells from mouse glomeruli. Kidney Int 2004, 65(6):2223-2227.

7. Demeule M, Labelle M, Regina A, Berthelet F, Beliveau R: Isolation of endothelial cells from brain, lung, and kidney: expression of the multidrug resistance P-glycoprotein isoforms. Biochem Biophys Res Commun 2001, 281(3):827-834.

8. McGinn S, Poronnik P, Gallery ED, Pollock CA: A method for the isolation of glomerular and tubulointerstitial endothelial cells and a comparison of characteristics with the human umbilical vein endothelial cell model Nephrology (Carlton) 2004, 9(4):229-237.

9. Rops AL, van der Vlag J, Jacobs CW, Dijkman HB, Lensen JF, Wijnhoven TJ, van den Heuvel LP, van Kuppevelt TH, Berden $\mathrm{JH}$ : Isolation and characterization of conditionally immortalized mouse glomerular endothelial cell lines. Kidney Int 2004, 66(6):2193-2201.

10. Tasnim F, Zink D: Cross talk between primary human renal tubular cells and endothelial cells in cocultures. Am J Physiol Renal Physiol 2012, 302(8):F1055-F1062.

11. Doctor RB, Chen J, Peters LL, Lux SE, Mandel LJ: Distribution of epithelial ankyrin (Ank3) spliceoforms in renal proximal and distal tubules. Am J Physiol 1998, 274(1 Pt 2):F129-F138.

12. Marelli-Berg FM, Peek E, Lidington EA, Stauss HJ, Lechler RI: Isolation of endothelial cells from murine tissue. J Immunol Methods 2000 244(1-2):205-215.

13. Ichimura K, Stan RV, Kurihara H, Sakai T: Glomerular endothelial cells form diaphragms during development and pathologic conditions. J Am SoC Nephrol 2008, 19(8):1463-1471.

14. Diehl L, Schurich A, Grochtmann R, Hegenbarth S, Chen L, Knolle PA: Tolerogenic maturation of liver sinusoidal endothelial cells promotes B7-homolog 1-dependent CD8+ T cell tolerance. Hepatology 2008, 47(1):296-305.

15. Tan TK, Zheng G, Hsu TT, Wang Y, Lee WW, Tian X, Cao Q, Harris DC: Macrophage matrix metalloproteinase- 9 mediates epithelial-mesenchymal transition in vitro in murine renal tubular cells. Am J Pathol 2010, 176(3):1256-1270.

16. Humphreys BD, Lin SL, Kobayashi A, Hudson TE, Nowlin BT, Bonventre JV, Valerius MT, McMahon AP, Duffield JS: Fate tracing reveals the pericyte and not epithelial origin of myofibroblasts in kidney fibrosis. Am J Pathol 2010, 176(1):85-97.

17. Ferrara N: Vascular endothelial growth factor. Arterioscler Thromb Vasc Biol 2009, 29(6):789-791.

18. Schrijvers BF, Flyvbjerg A, De Vriese AS: The role of vascular endothelial growth factor (VEGF) in renal pathophysiology. Kidney Int 2004, 65(6):2003-2017

19. Nakamura T, Mizuno S: The discovery of hepatocyte growth factor (HGF) and its significance for cell biology, life sciences and clinical medicine. Proc Jpn Acad Ser B Phys Bio/ Sci 2010, 86(6):588-610.

20. You WK, McDonald DM: The hepatocyte growth factor/c-Met signaling pathway as a therapeutic target to inhibit angiogenesis. BMB Rep 2008, 41(12):833-839.

\section{doi:10.1186/s12860-014-0040-6}

Cite this article as: Zhao et al:: Isolation and epithelial co-culture of mouse renal peritubular endothelial cells. BMC Cell Biology 2014 15:40.

\section{Submit your next manuscript to BioMed Central and take full advantage of:}

- Convenient online submission

- Thorough peer review

- No space constraints or color figure charges

- Immediate publication on acceptance

- Inclusion in PubMed, CAS, Scopus and Google Scholar

- Research which is freely available for redistribution 